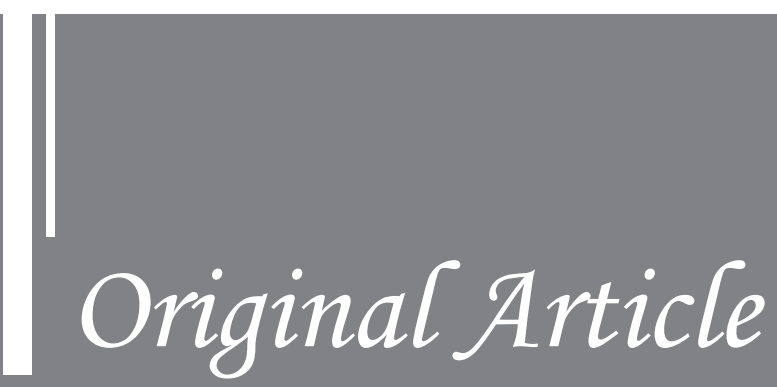

\title{
ACUTE FLACCID PARALYSIS SURVEILLANCE SYSTEM: CURRENT STATUS IN AZAD JAMMU \& KASHMIR- 2018
}

\author{
Syed Nadeem Ur Rehman', Uzma Hafeez', Mumtaz Ahmad Khan², \\ Syed Masood Ahmad Bukhari ${ }^{1}$ Kavita $^{3}$
}

\author{
${ }^{1}$ Directorate General Health, Muzaffarabad AJK \\ ${ }^{2}$ AJK Medical College Muzaffarabad AJK \\ ${ }^{3}$ Civil Hospital Mirpurkhas
}

Correspondence: Syed Nadeem Ur Rehman. Email: drnadeemsyed999@gmail.com

\begin{abstract}
Background: The State of Azad Jammu \& Kashmir (AJ\&K) is polio free since October 2000. The objectives of our study is to review of existing Acute Flaccid Paralysis Surveillance System in Azad Jammu \&Kashmir, identify the strong \& weak points of the existing system and suggest course of action for efficient performance of the existing system.

Methods: This qualitative \& quantitative evaluation was conducted at Provincial Disease Surveillance \&Response Unit (PDSRU) Muzaffarabad Azad Jammu \& Kashmir during March -April 2019. The database of AFP cases during 2018 was reviewed and relevant stakeholder's interviews were conducted consulting guidelines formulated by the Centre for Disease Control \& prevention(CDC) in 2001 for Evaluating Public Health Surveillance Systems.

Results: In 2018, a total of 265 AFP cases were registered. The mean age was 65 months (range $01-180$ months). 59\% (n=157) were male children. $58 \%$ of cases were under 05 year's age. Standardized case definition and data format with simple information flow was found. System was flexible enough to incorporate measles and neonatal tetanus cases since 2009. Data quality was excellent (100\% zero and monthly reports). A close coordination was observed amongst all relevant stakeholders. Sensitivity was $200 \%$. No polio case was identified and therefore, PPV was zero. Majority of cases were reported by public sector (93\%).Sufficient financial as well as skilled human resources were available and hence system found stable. Timeliness of reporting found $90 \%$.

Conclusion: The performance of AFP surveillance system in AJ\&K is up to the mark. However, there is constant threat of reintroduction of polio virus from adjacent area of Punjab \& Khyber Pakhtunkhwa provinces. Highly vigilant AFP surveillance system with capacity of rapid response is the solution. Furthermore, it is vital to sustain the AFP Surveillance till the goal of global polio eradication is achieved. Keywords: AFP in AJ\&K, surveillance system, global polio eradication
\end{abstract}




\section{Introduction}

The term acute flaccid paralysis (AFP) may be defined as acute onset of flaccid paralysis, in a child belonging to either sex and having age less than 15 years, in one or more limbs due to the neurological abnormality caused by poliomyelitis, meningitis, encephalitis, traumatic neuritis, transverse myelitis, Guillain-Barre syndrome or brain tumors (1) The AFP cases in polio non vaccinated children of age less than 5 years who presented with fever and weakness in one or more limbs are the potential polio cases and need further investigation to rule out poliomyelitis.(2)

Public health surveillance may be defined as the systematic collection, analysis and interpretation of health-related data and its dissemination to the relevant stakeholders with ultimate goal of reduction in morbidity and mortality.(3) The systematic analysis of functioning of a surveillance system according to predetermined objectives is called evaluation of a surveillance system.(4) The ultimate goal of AFP surveillance is cessation of poliovirus circulation in a given country.(5) Acute flaccid paralysis surveillance is considered as an essential strategy in global eradication of polio. The objectives of our study is to review of existing Acute Flaccid Paralysis Surveillance System in Azad Jammu \&Kashmir, identify the strong \& weak points of the existing system and suggest course of action for efficient performance of the existing system.

\section{Methodology}

A clinical AFP case is defined by WHO as "any child under 15 years of age with Acute Flaccid Paralysis or any person of either sex and of any age who presented with muscles weakness and the clinician suspects poliomyelitis" while a confirmed case is a clinical case with laboratory confirmation of wild poliovirus in Azad Jammu \& Kashmir from 01st January 2018 to 31 st December 2018. Database of AFP surveillance 2018 maintained by WHO regional Office at Muzaffarabad was analyzed at PDSRU Muzaffarabad using guidelines formulated by Centre for Disease Control and Prevention(CDC) in 2001 for Evaluating Public Health Surveillance Systems (6) The present study was conducted during March 2019. The evaluation process was conducted at three levels, regional (Directorate), intermediate (Districts) and local level (first level care facilities) and EPI department of Abbas Institute of Medical Sciences (AIMS) Muzaffarabad. Focal points personnel, active surveillance personnel, district health managers were interviewed in addition to pediatricians, public health specialists, general practitioners, medical and laboratory technicians to evaluate their knowledge about the surveillance system according to the WHO field guidelines. All the relevant records of the active and passive surveillance were observed and evaluated for the year 2018. All the performance indicators were calculated and compared with the international targets.

Results

The qualitative and quantitative evaluation of functioning of AFP surveillance system in State of Azad Jammu \& Kashmir is as follows:

Simplicity

The term simplicity may be defined as easiness in structure as well as operation of the given surveillance system. (6)Salient features of AFP surveillance system for this attribute are as follows:

o Uniform case definition of AFP case developed by WHO is used which is uncomplicated and easily understandable.

o Data format \& information flow simple

o Polio conformation requires wild polio virus isolation from stool specimen by WHO accredited virology laboratory which is based in National Institute of Health Islamabad.

o Investigation of AFP case requires home visit by the relevant team for gathering relevant data and collection of stool specimens.

o Reporting of AFP cases was found at many levels (district, province, country, EMRO and WHO HQ at Geneva)

\section{Flexibility:}

The term flexibility is defined as ability of operational adjustment of a surveillance system with minimal financial and human recourses. (6)Salient features of AFP surveillance system for this attribute are as follows:

" The system is flexible as it is capable to adopt changes e.g., measles \& neonatal tetanus reporting since 2009.

Data Quality:

The data quality is defined as completeness of dataset of a given surveillance system. (6) Salient features of AFP surveillance system for this attribute are as follows:

o Completeness of data verified at District Health Officers' office \& Provincial Program Manager office ( $100 \%$ weekly zero reports and monthly reports from all reporting sites)

\section{Acceptability:}

The term acceptability is defined as keenness of all relevant stakeholders for contribution in the given surveillance system.(6) Salient features of AFP surveillance system for this attribute are as follows:

o All stakeholders of AFP surveillance system work in liaison and close coordination with each other.

o Well maintained record of AFP surveillance at District Health Officers' office, Provincial Program Managers' office and WHO office.

Sensitivity

The term sensitivity is defined as the ability to accurately identify what is supposed to gauge of a given surveillance system. (6) Salient features ofAFP surveillance system for this attribute are as follows:

o The total projected population of children less than 15 years in AJ\&K during 2018 according to WHO and Health department record is $21,57,747$.

o WHO target rate of AFP surveillance for endemic countries is 6/100000 and hence the target is 132 cases.

o The sensitivity of the surveillance system is $253 / 132 * 100$ $=200.7 \%$. Highly sensitive AFP surveillance system is critical for not missing even "single case of polio".

o The AFP surveillance system has ability to detect epidemics which in case of polio is detection of single case.

\section{Predictive Value Positive}

The term Predictive Value Positive (PVP) is defined as the proportion of the true cases out of the total cases reported of a given surveillance system.(6) Salient features of AFP surveillance system for this attribute are as follows:

o No polio case was reported in AJ\&K during 2018 and hence the PPV is zero.

\section{Representativeness}

The term representativeness is defined as description of the cases with respect to time place and person of a given surveillance system.(6) Salient features of AFP surveillance system for this attribute are as follows:

o A total of 265 cases of AFP were reported during 2018 in AJ\&K.

o $\quad 157$ cases $(59 \%)$ were male children and 108 cases $(41 \%)$ were female children.

o Age of AFP cases ranges from one month to 15 years

o No of cases age less than 1 year were 11( $4.1 \%), 1-5$ years were $143(54.0 \%), 6-10$ years were $72(27.1 \%)$ and from $11-15$ years were 39 cases $(14.8 \%)$

o The most common diagnosis was Guillain-Barre syndrome .151 cases $(57 \%)$ followed by Transverse myelitis 48 cases $(18 \%)$ and encephalitis 37 cases $(14 \%)$

o More cases were reported during winter season as compared to summer.

o Highest number of cases were reported from district Poonch $(n=44)$ followed by district Kotli and district Bagh

o AFP surveillance system covered all public primary secondary and tertiary healthcare facilities and key physicians in private sector across the whole territory of AJ\&K. 244 cases of $\operatorname{AFP}(92 \%)$ were registered in public health facilities 13 cases $(5 \%)$ by armed forces health facilities in AJ\&K and eight cases were $(3 \%)$ reported by private practitioners.

o No case was classified as acute poliomyelitis in AJ\&K during 2018

Stability

The term stability is defined as consistence of dataflow in the surveillance system.(6)Salient features of AFP surveillance system for this attribute are as follows:

o Well trained workforce and infrastructure

o Availability of funds for AFP surveillance

o Computerized data entry and communication at districts \& state levels

Timeliness

The term timeliness is defined as proportion of cases who fulfill pre determined timelines.(6). The timely reporting of AFP cases is critical for prompt diagnosis and control of poliomyelitis. Timeliness of AFP 
surveillance was found 90\% during 2018 .

\section{Usefulnes}

The term usefulness of AFP surveillance system is defined as its ability to prevent and control poliomyelitis by timely detection and response. In this regard, AFP surveillance system is quite useful as it has met consistently its objective of prevention and control of poliomyelitis in the State of Azad Jammu \& Kashmir which is polio free since 2000 Discussion

The AFP surveillance remained the fundamental approach for polio eradication worldwide. In Pakistan, AFP surveillance system had been organized at multiple levels (national, provincial, district and tehsil).AFP surveillance had been started in Azad Jammu \& Kashmir in 1995 with the goal to eliminate all cases of polio.(7) Our study showed that AFP surveillance is simple, swift, sensitive, flexible and well organized in Azad Jammu \&Kashmir AJ\&K) in contrast to AFP surveillance system in Zimbabwe and Launda which is complex and less sensitive. $(8,9)$ All the stake holders knew the objectives of AFP surveillance and the system was functioning according to WHO field guidelines in AJ\&K.(10) The role of AFP surveillance in making country polio free has been highlighted in many studies carried out across the globe. $(11,12,13,14,15)$

Our study has following limitations namely less time been available for stakeholders' interviews therefore selected interviews were conducted. Less resources and logistics were available, therefore selected AFP surveillance reporting sites were visited in district Muzaffarabad only. Record of AFP Surveillance was observed only at PPM EPI Office, DHO Office Muzaffarabad, WHO Regional Office Muzaffarabad and EPI vaccination centre at Abbas Institute of Medical Sciences Muzaffarabad due to time and financial constraints.

\section{Conclusion}

The performance of AFP surveillance system in AJ\&K is up to the mark set by World Health Organization. However, there is space for further improvement due to threat of reintroduction of polio virus from nebouring areas of Punjab \& Khyber Pakhtunkhwa provinces. Highly vigilant AFP surveillance system with capacity of rapid response is the solution. Furthermore, it is vital to sustain the AFP Surveillance till the goal of global polio eradication is achieved

\section{References}

1. Ashraf S, Rahman AJ. Guillain Barre Syndrome; Clinical feature in children. Professional Med J 2013;20(3): 348-353

2. Rufaro MC, Kumar SB, Seter S. Performance of acute flaccid paralysis surveillance system in Zambia: 2000 to 2009-Analysis of secondary data International Journal of Public Health and Epidemiology. 2014: $10(3): 75-81$
3. Pencheon D, Guest C, Melzer D, Gray JAM. Scoping public health problems, Oxford Handbook of Public Health Practice, Second edition, Oxford University Press, Oxford: 2006:4-11.

4. Porta M. A Dictionary of Epidemiology. 5th edition. New York, NY: Oxford University Press; 2009.

5. World Health Organization. Guidelines for preparedness and response for wild poliovirus importation and format for national plans

6. Updated guidelines for evaluating public health surveillance systems. Recommendations from the Guidelines Working Group. Centers for Disease Control and Prevention, Atlanta USA.

7. Muhammad S, Iqbal H, Fahad A. Audit and evaluation of the acute flaccid paralysis surveillance system in Khyber Pakhtunkhwa,Pakistan. KMUJ :2016, 8(1):22-26

8. Kufakwanguzvarova Pomerai, Robert F Mudyiradima, Mfuta Tshimanga, and Mary Muchekeza. Evaluation of the Acute Flacid Paralysis (AFP) Surveillance System in Bikita District Masvingo Province 2010. BMC Res Notes 2014;7:252.

9. Macama A, Okeibunor J, Grando S, Djibaoui K, Yameogo RK, Morais A, et al. Reasons and circumstances for the late notification of Acute Flaccid Paralysis (AFP) cases in health facilities in Luanda. Pan Afr Med J 2014 ; 18:239-40.

10. World Health Organization .Global Polio Eradication Initiative, Jan. 2013

11. Lam RMK, Tsang THF, Lim WL. Surveillance of acute flaccid paralysis in Hong Kong:1997-2002.Hong Kong Med J.2005:11(3)164-173

12. Odoom JK, Ntim NA, Sarkodie B, Addo J, Minta-Asare K, Obodai E, et al. Evaluation of AFP surveillance indicators in polio-free Ghana, 2009-2013. BMC Public Health 2014 Jul 5; 14:687.

13. Kufakwanguzvarova Pomerai, Robert F Mudyiradima, Mfuta Tshimanga, and Mary Muchekeza. Evaluation of the Acute Flacid Paralysis (AFP) Surveillance System in Bikita District Masvingo Province 2010. BMC Res Notes 2014; 7: 252-53.

14. Bassey BE, Gasasira A, Mitula P, Frankson UU, Adeniji JA. Surveillance of acute flaccid paralysis in Akwa Ibom State, Nigeria 2004-2009. Pan Afr Med J. 2011; 9:32.

15. .John TJ, Vashishtha VP. Eradicating poliomyelitis: India's journey from hyper endemic to polio-free status. Indian J Med Res 2013; 137(5):881-94. 